\title{
Conclusions
}

Plusieurs applications peuvent se concevoir :

$1^{\circ}$ Dans le domaine du laboratoire, on peut étudier la mise au point d'une méthode de dosage biologique de la caséine et de la lactalbumine dans les fromages en particulier. De premiers essais ont été encourageants puisqu'ils nous ont permis de classer des fromages spécialement préparés avec des pourcentages variables de lactalbumine; des échantillons dans lesquels a vaient été incorporées des doses de $5,10,15$ et $20 \%$ d'albumine, ont été ensemencés puis mis à l'étuve à $30^{\mathrm{e}}$. Les pourcentages d'acides a minés obtenus ont été inversement proportionnels à la quantité d'albumine incorporée ;

$2^{\circ}$ Dans le domaine industriel, plusieurs applications sont envisàgées :

a) Fabrication de produits a minés à partir de la caséine, pour l'alimentation humaine et animale.

b) Utilisation de cette dégradation de la caséine dans la maturation de certains fromages. Des essais, entrepris dans la fabrication des pâtes molles, ont permis d'améliorer nettement la qualité des produits, en procédant par ensemencement de levain de mycoderme dans le lait de baquettes. Les fromages obtenus a vec ensemencement de cultures de myeoderme présentaient une onctuosité plus grande de la pâte et une maturation à froid accélérée.

D'autres essais sont entrepris actuellement sur des fromages à pâte pressée (Saint-Paulin).

(Travail de la Station Centrale de Technologie Agricole)

\section{CONTRIBUTION A LA CONNAISSANCE DU BEURRE DE BREBIS (BEURRE DE CASHCAVAL) PRÉPARÉ EN ROUMANIE}

\author{
par \\ le Dr C. STOIAN
}

de l'Institut National Zootechnique de Bucarest

Le lait de brebis est employé presque exclusivement à la préparation des fromages, qui sont particulièrement appréciés dans tous les pays.

La préparation du beurre de lait de brebis, dans les mêmes conditions que celui du lait de vache, est tout à fait sporadique et en quantités extrêmement réduites ; rien que pour les besoins du personnel de la bergerie, et cela surtout dans les pays du sud-est de l'Europe.

Ayant en vue cette production réduite, nous ne trouverons pas 
d'études nombreuses sur la constitution de ce beurre. Par contre, il y a des études sur la matière grasse du lait de brebis, pour établir sa composition, en rapport avec celle du lait de vache, ou de chèvre, parce que, souvent, ces deux dernières catégories de lait sont utilisées pour la falsification de la première; pour ceci les divers auteurs ont étudié la matière grasse du lait de brebis et en ont déterminé les constantes physiques et ehimiques.

En étudiant la littérature nous voyons que les auteurs n'ont jamais déterminé, sur les mêmes échantillons, toutes les constantes. Certains chercheurs ont déterminé seulement une des constantes.

Ainsi, Pezzr [1] a établi le point de fusion, $29^{\circ} \mathrm{C}$. ; le point de solidification, $12^{\circ} \mathrm{C}$. ; l'indice de Reichert-Meissl, 32,9.

Petkow [2] a étudié le beurre de brebis préparé en Bulgarie, dans des conditions proches de la préparation du beurre de lait de vache, et a établi les chiffres suivants :

Poids spécifique à $100^{\circ} \mathrm{C}$. : 0,8693 ; degré réfractométrique à $40^{\circ}$ C. : $44^{\circ} 4$; indice de saponification : 227,8 ; indice Reichert-Meissl : 26,8 ; indice d'iode: 35,14 ; acides gras solubles: 88,5 ; acidité libre: 2,01 .

Martin [3] détermine un certain nombre d'indices sur 26 échantillons de beurre de lait de brebis, de la région de Roquefort, préparé dans le laboratoire et constate :

Indice de réfraction : 1045215 ; indice de Crismer : $54084 \mathrm{C}$.; indice de saponification : 231,58 ; indice de Reichert-Meisl : 28,48 ; indice de Polenske: 4,4, avee une variation de 2,53 à 7,01 ; indice de Planchon : 23,6 ; acide butyrique $\%: 12,05$.

Sснмітт [4] détermine seulement l'indice de Hehner et il l'établit à 85,3 .

Fascetti et SAvini [5] constatent que pour l'identification des falsifications du fromage italien «Pecorino romano ", qui est préparé exclusivement de lait de brebis, il suffit de déterminer l'indice de Polenske sur la matière grasse du fromage, et ils établissent cet indice à une valeur qui varie de 4 à 6 . Ces auteurs croient que l'indice de Polenske constitue le seul moyen d'établir les falsifications.

Du même avis que Fascetri et Savini sont aussi les auteurs américains I. D. GARARD et D. PASCALE [11] qui étudient la matière grasse du fromage de Roquefort et arrivent à la conclusion que l'indice de Polenske varie de 4 à. 8 , pendant que le même indice, pour le lait de vache, a une valeur comprise entre 1,5 et 3 .

DaRMoIs [7] constate que dans le beurre de vache, l'indice de Polenske et l'indice de Reichert-Meissl varient peu et dans le même 
sens, tandis que pour le beurre de brebis, l'indice Reichert-Meissl croît peu, jusqu'à 33 , mais l'indice de Polenske arrive même à 8 .

Chollet et Camus [9] ont étudié en France la graisse du lait de chèvre et ont persisté toujours sur les indices de Polenske et de Reichert-Meissl, en constatant que l'indice Polenske varie de 5 à 9,7 et l'indice de Reichert-Meissl de 16 à 28,2 . En même temps, ces auteurs établissent la relation suivante entre les acides volatils solubles et insolubles, pour la graisse du lait de chèvre :

$$
\frac{\text { Acides volatils insolubles (A. V. I.) } \times 100}{\text { Acides volatils solubles (A. V. S.) }}=22,8 \text { à } 41,4
$$

tandis que pour la graisse du lait de vache cette relation a une valeur qui varie de 8 à 15 .

- La matière grasse du lait de chèvre a été étudiée aussi par C. P. Lopez [10], en Espagne, toujours en vue d'établir une méthode de contrôle pour lesfalsifications du lait de vache avec du lait de chèvre. L'auteur arrive à la même conclusion, c'est-à-dire que l'indice de Polenske constitue le seul moyen de contrôle.

G. GÉNIN [14] a fait une revue sur les recherches entreprises par les divers auteurs sur les falsifications du fromage de Roquefort et est arrivé à la conclusion que les indices de Reichert-Meissl et de Polenske sont les seuls indiqués pour nous montrer s'il est question de falsification ou non.

La valeur des indices de la matière grasse du lait de brebis est citée aussi par d'autres auteurs, comme : A. Bömer et I. Grossfeld [12], W. Halden et A. Grün [13], Rochaix et Tapernoux [15]. Les chiffres indiqués par ces auteurs concordent avec ce qui a été dit plus haut.

Pour synthétiser les résultats indiqués par les différents auteurs j'ai fait le tableau I, ci-contre.

\section{$*^{*} *$}

Sur le beurre de brebis préparé en Roumanie ne se sont pas effectuées des recherches jusqu'à présent; par ailleurs, ce beurre est préparé dans des conditions différentes de celui qui a servi aux recherches eitées plus haut. Nous avons, dans ces conditions, trouvé nécessaire de faire ce travail, surtout si nous avons en vue qu'en Roumanie on prépare chaque année de 15 à 20 wagons de beurre de brebis.

En Roumanie, sous le nom de beurre de brebis on entend, en général, le beurre fabriqué de la matière grasse résultant de l'échaudage du caillé pendant la fabrication du fromage de "cashoaval ". C'est pour cela que le beurre de brebis est connu encore sous le 
TABLEAU.I

RÉSULTATS OBTENUS ET GITÊS PAR LES DIVERS AUTEURS SUR LES INDIGES DE LA MATIERE GRASSE DU LAIT DE BREBIS

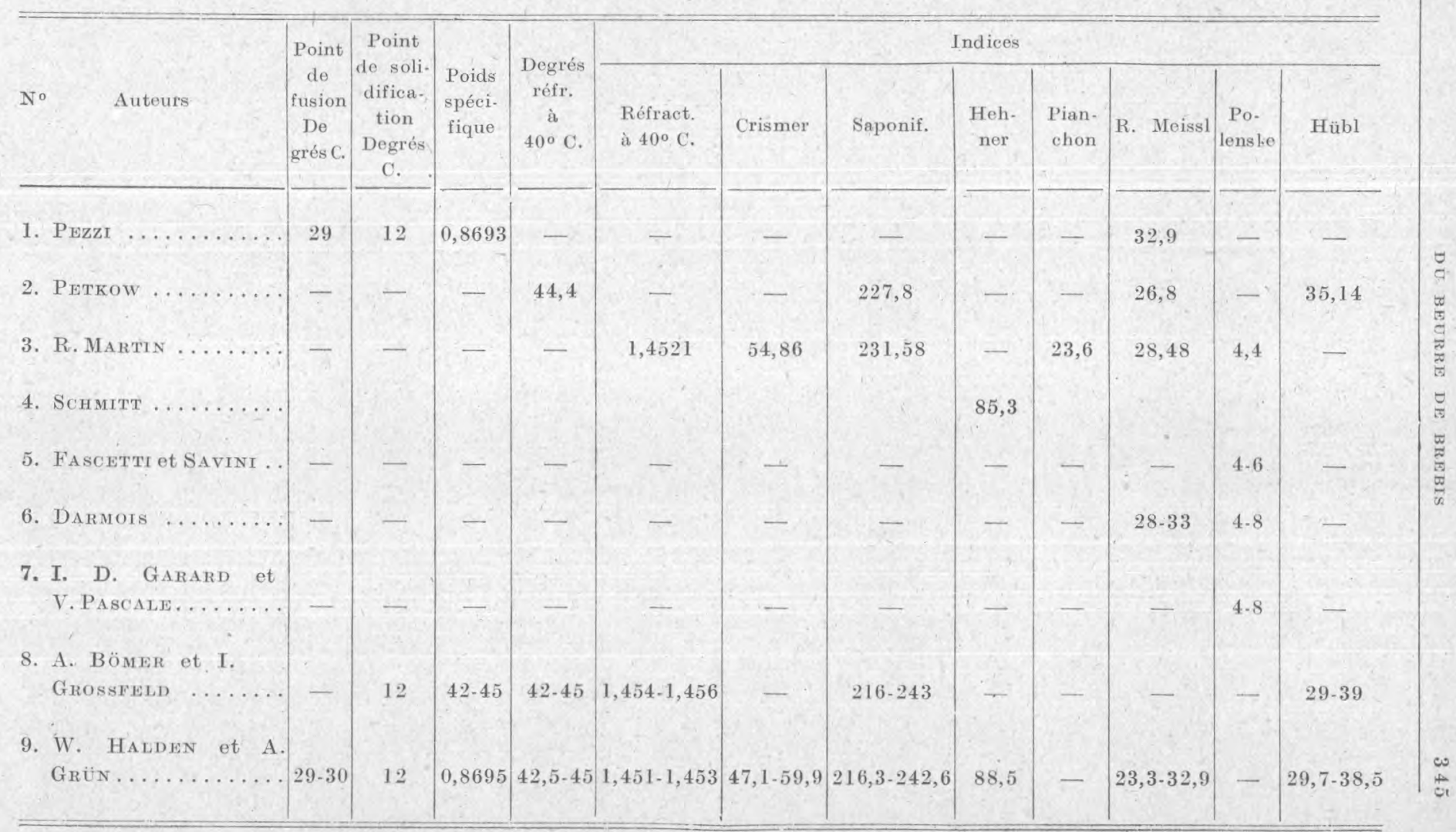


nom de beurre de "cashcaval ". Mais, nous trouvons qu'il serait logique de le nommer beurre fondu de brebis, car, quelles que soient les conditions de la fabrication, il n'est mis dans le commerce que sous la forme de beurre fondu.

\section{Fabrication du beurre de cashcaval}

A. 1. La plus grande partie du beurre de cashcaval est préparée comme il suit : lorsque le caillé de brebis est suffisamment fermenté pour la fabrication du cashcaval, ce caillé est coupé en tranches que l'on met dans des paniers en bois, ou en métal, qui sont introduits dans des cuves avec de l'eau chauffée à $80^{\circ} 90^{\circ}$ C. Ces paniers sont - bien agités, jusqu'à ce que les tranches de caillé forment une pâte uniforme.

Dans certaines régions, surtout là où on prépare du cashcaval mou (dit de Penteleu), les tranches de caillé sont introduites directement dans l'eau de la cuve, sans qu'elles soient au préalable mises dans les paniers spéciaux.

2. Pendant l'agitation et le pétrissage du caillé dans l'eau bouillante, une partie de la matière grasse fond et surnage à la surface de l'eau. Cette graisse est ensuite, enlevée, avec une certaine quantité d'eau et est introduite dans des tonneaux en bois où l'eau se sépare au fond, d'où elle est éliminée par une cannelle placée à la partie inférieure du tonneau. Ainsi, on ajoute, journellement, la graisse résultant de l'échaudage du eaillé sur la graisse déjà existante dans le tonneau, obtenue dans les mêmes conditions, jusqu'à ce que le tonneau soit à moitié rempli, ce qui dure, en moyenne, près d'une semaine.

3. Lorsque le tonneau a été à moitié rempli, le beurre est baratté avec une pièce spéciale en bois. Le beurre est obtenu après $1 / 2-1$ heure de barattage.

4. Après le barattage on fait sortir du tonneau le beurre, qui est placé dans un autre tonneau en bois, à la température de la chambre, où il reste jusqu'à ce qu'on obtienne une quantité suffisante pour être fondue.

Ainsi, certaines quantités restent à la température de $20-30^{\circ} \mathrm{C}$. et à la lumière, pendant douze à vingt jours.

5. Quand on a une quantité suffisante de beurre, celui-ci est mis dans des cuves métalliques et chauffé au feu, à l'ébullition, pendant trois à quatre heures. Pendant l'ébullition, la plus grande partie de l'eau s'évapore et il ne reste plus, sous la matière grasse, au fond de la cuve, qu'une petite quantité d'eau, qui avec d'autres substances non grasses (caséine, lactose, sels minéraux), forme ce qu'on appelle " l'azure de beurre ". 
6. Après la fonte et le refroidissement, le beurre fondu est mis dans des bidons métalliques, sans être au préalable mélangé, bien qu'il soit constaté qu'il se sépare en deux couches de consistance différente : une couche supérieure plus fluide que la couche inférieure.

Le beurre de brebis (beurre de cashcaval) ainsi préparé est connu aussi sous le nom de beurre bouilli.

B. Un autre procédé pour la préparation du beurre de brebis est le suivant :

Dès que le lait est caillé et le coagulum broyé, on ramasse la caillebote qui est bien pressée; il en résulte une quantité de sérum opalescent, riche en matière grasse, qui est passé au séparateur et on obtient ainsi une erème qui reste un à deux jours à la fermentation, puis la crème est barattée. Le beurre obtenu est fondu dans une cuve au soleil, sur un faible feu. Pendant sa fonte, le beurre est bien mélangé à la main, júsqu'à ce qu'il soit complètement limpide, puis le beurre fondu est retiré du feu et laissé une à deux heures pour la séparation de-l'eau. Ensuite, le beurre est versé dans des bidons de 18 à 20 kilogrammes. Le beurre ainsi préparé est nommé "Muraitcu " il est préparé surtout en Dobroudja par les roumains macédoniens.

C. Enfin, il y a des bergers qui ramassent la matière grasse résultant de l'échaudage de la caillebote, pendant toute la période de fabrication, la déposent dans des grands tonneaux, où elle est, de temps en temps mélangée en lui ajoutant des petites quantités de sel, pour éviter toute odeur, puis, en automne, vers septembre, ils fondent le beurre et le mettent dans des bidons.

De tout ceci, on constate que le beurre de brebis ne se prépare pas dans les mêmes conditions que le beurre de vache, de la crème extraite du lait, mais, au contraire, le beurre de brebis est un produit secondaire qui résulte de la fabrication du fromage de "cashcaval ".

La plus importante région où l'on prépare le beurre de brebis (beurre de cashcaval) est la Dobroudja, puis certains départements de la plaine du Danube, ainsi que la-région de montagne des départements de Buzau et de Prahova, où l'on fabrique du cashcaval de "Penteleu ». Des quantités infimes sont préparées aussi dans les départements de Nasaud et de Maramuresh.

\section{Bref coup d'œil sur l'exécution de nos analyses}

Nous avons analysé, au total, huit échantillons de beurre de brebis, échantillons pris sur le marché de la ville de Bucarest. Tous les échantillons proviennent de la fabrication de 1945, tandis que les analyses ont été exécutées dans la période septembre 1945 - 
mars 1946. Nous avons pris nos échantillons seulement des bidons en métal, pleins et bien fermés. Avant la prise des échantillons, le contenu des bidons était bien homogénéisé. On a pris pour chaque échantillon $500 \mathrm{gr}$. de beurre.

Les analyses de beurre de cashcaval sont réduites à l'analyse de la matière grasse, car le beurre de cashcaval, examiné par nous, ne contenait point de substances non grasses. Seulement, dans l'échantillon no 7 nous avons trouvé du $\mathrm{NaCl}(0,11 \%)$.

En ce qui concerne l'exécution proprement dite de nos analyses, nous remarquons les faits suivants :

Les points de fusion et de solidification ont été déterminés avec le tube capillaire annexé au thermomètre gradué à $1 / 20^{e}$;

Les acides solubles totaux (Planchon) et insolubles totaux (Hehner) ont été déterminés dans la solution alcoolique de $\mathbf{K O H}$, neutralisée avec $\mathrm{HCl}-\mathrm{N} / 2$, après la détermination de l'indice de saponification ;

L'acidité a été exprimée dans le chiffre de l'acidité ;

Le poids spécifique a été déterminé au picnomètre, à $15^{\circ} \mathrm{C}$. ; L'indice de réfraction a été déterminé à $40^{\circ} \mathrm{C}$.

\section{Discussion des résultats}

\section{A. Les constantes physiques.}

1. Les points de fusion et de solidification présentent des variations plus grandes que celles établies par d'autres auteurs. Ces variations gardent un certain rapport entre le point de fusion et celui de la solidification. L'échantillon n $\mathrm{n}^{\circ} 7$ a comme point de fusion : $19{ }^{\circ} \mathrm{C}$., et comme point de solidification : $9^{\circ} \mathrm{C}$., ce qui est au-dessous de la limite établie jusqu'à présent. Ceci est dô, à notre avis, au fait que le beurre de brebis (beurre de cashcaval) n'est point homogénéisé avant d'être versé dans les bidons, mais il est enlevé couche par couche, et il est constaté que les couches supérieures ont une consistance plus fluide que les couches inférieures, de telle façon que les bidons qui sont remplis les premiers contiendront du beurre avec points de fusion et de solidification inférieurs à ceux remplis à la fin de l'opération.

Nous avons constaté ce fait aussi dans notre laboratoire, car nous avons pris de chacun des échantillons, $100 \mathrm{~cm}^{3}$ de beurre fondu et bien homogénéisé, dans un tube de verre cylindrique et gradué; puis le beurre était laissé à la température de la chambre (200 C.) ; . après 24 heures, il s'est séparé en deux couches : celle supérieure 
TABLEAU II

RÉSULTATS DE NOS ANALYSES SUR LES 8 ÉGHANTILLONS DE BEURRE DE CASHCAVAL (BEURRE DE LAIT DE BREBIS).

\begin{tabular}{|c|c|c|c|c|c|c|c|c|c|c|c|}
\hline \multirow{2}{*}{ Indices } & \multicolumn{8}{|c|}{ Echantillons } & \multicolumn{3}{|c|}{ Valeur } \\
\hline & 1 & 2 & 3 & 4 & 5 & 6 & 7 & 8 & Maxima & Minima & Moyenne \\
\hline Pointdefusion $\ldots \ldots \ldots \ldots \ldots \ldots$ & $19 \circ 3$ & $28^{\circ}$ & 2902 & $24^{\circ} 8$ & $31 \circ 7$ & 2807 & 2809 & 2902 & 3107 & $19^{\circ} 3$ & $27 \circ 6$ \\
\hline Point de solidification......... & $9^{\circ}$ & $13^{\circ} 3$ & $12 \circ 7$ & $10^{\circ} 7$ & $14^{\circ} 8$ & $12 \circ 9$ & $13^{\circ} 1$ & $12^{\circ} 8$ & $14^{\circ} 8$ & $9^{\circ}$ & $11^{\circ} 04$ \\
\hline Poids spécifique $\ldots \ldots \ldots \ldots \ldots$ & 0,9246 & 0,9249 & 0,9244 & 0,9259 & 0,9259 & 0,9252 & 0,9249 & 0,9246 & 0,9259 & 0,9246 & 0,92505 \\
\hline Indice de réfraction à $40^{\circ} \mathrm{C} \ldots \ldots$ & 1046.127 & 1046103 & 1046103 & $1^{\circ} 46097$ & 1046103 & 1046103 & 1046097 & 1046103 & 1046127 & 1046103 & 1046104 \\
\hline Degré réfractométrique........ & 44,7 & 44,3 & 44,3 & 44,2 & 44,3 & 44,3 & 44,2 & 44,3 & 44,7 & 44,2 & 44,325 \\
\hline Indice de Crismer $\ldots \ldots \ldots \ldots \ldots$ & $56^{\circ 4}$ & $56^{\circ} 16$ & $58^{\circ} 04$ & $54^{\circ}$ & $55^{\circ} 2$ & $56^{\circ}$ & $56^{\circ} 23$ & $55^{\circ} 8$ & $58^{\circ} 04$ & $55^{\circ} 2$ & $55^{\circ} 73$ \\
\hline Indice de saponification $\ldots . .$. & 233,4 & 233,28 & 230,19 & 234,51 & 230,02 & 231,12 & 231,148 & 232,16 & 233,4 & 230,19 & 231,97 \\
\hline Indice de Hehner. . . . . . . . . . & 86,19 & 85,46 & 83,76 & 86,14 & 85,88 & 86,28 & 86,43 & 86,9 & 86,9 & 85,46 & 85,88 \\
\hline Indice de Planchon $\ldots . . . .$. & 33,32 & 35,61 & 34 & 33,1 & 32,6 & 33,5 & 32,78 & 32,75 & 35,61 & 32,75 & 33,46 \\
\hline Indice deReichert-Meiss] $\ldots . .$. & 31,57 & 29,2 & 30,4 & 30,6 & 27,43 & 28,6 & 30,75 & 30,48 & 31,57 & 27,43 & 29,88 \\
\hline Indice de Polenske . . . . . . . . . & 4,25 & 5,3 & 4,35 & 5,25 & 4,3 & 4,6 & 4,8 & 4,9 & 5,3 & 4,25 & 4,53 \\
\hline Indice de Hubl . ............ & 41,168 & 40,74 & 40,52 & 38,07 & 36,512 & 38,88 & 37,39 & 39,16 & 41,168 & 37,39 & 39,43 \\
\hline Acidité $\ldots \ldots \ldots \ldots \ldots \ldots \ldots \ldots$ & 1,57 & 1,62 & 1,46 & 0,78 & 0,67 & 1,23 & 0,35 & 1,36 & 1,62 & 0,35 & 1,13 \\
\hline$\frac{\text { A. V.I. } \times 100}{\text { A. V. S. }}$ & 13,46 & 18,1 & 14,4 & 17,1 & 15,7 & 16,08 & 15,6 & 16,07 & 18,1 & 13,46 & 15,1 \\
\hline $\mathrm{NaCl}$ & & & & & & & $0,11 \%$ & & & & \\
\hline
\end{tabular}


encore liquide et transparente, celle inférieure, opaque et solidifiée.

La variation de la température de fusion et de solidification est ainsi due à la non-homogénéisation du beurre avant d'être versé dans les bidons.

2. Le poids spécifique, déterminé à $15^{\circ} \mathrm{C}$., varie entre des limites très étroites (0,9244-0,9259), a vec la moyenne de 0,92505 . Nos résultats sur le poids spécifique ne peuvent pas être comparés à ceux d'autres auteurs parce que ceux-là ont déterminé le poids spécifique à la température de $100^{\circ} \mathrm{C}$.

3. L'indice de réfraction est supérieur à celui établi antérieurement et varie de 1046103 à 1046127 , a vec la moyenne de 10461045 . En ce qui concerne le nombre de degrés réfractométriques, les résultats obtenus par nous s'encadrent entre les limites citées par quelques auteurs.

4. La température critique de dissolution (indice de Crismer) correspond aux données citées par W. Halden et A. Grün.

\section{B. Constantes chimiques.}

1. L'indice de saponification est supérieur à celui établi par quelques auteurs et s'encadre entre les limites établies par d'autres. D'après nos recherches, il est constaté que cet indice présente une variation très réduite $(230,19-233,4)$, pendant que les auteurs citent des variations beaucoup plus larges (216-243).

2. L'indice de Hehner, est en moyenne de 85-88 en se rapprochant des chiffres cités par Sснмттт, mais il est inférieur à celui cité par d'autres auteurs. En outre, sur l'indice de Hehner n'ont été faites, jusqu'à présent, que peu de recherches.

3. L'indice de Planchon est supérieur à celui établi pour le beurre de brebis préparé dans le laboratoire régional de Rodez, par Martin qui, lui, a trouvé la valeur moyenne de 23,6. Aucun autre auteur n'a plus déterminé la valeur de l'indice de Planehon pour la graisse du lait de brebis. La valeur de l'indice de Planchon établi par nous est en moyenne 33,457 ce qui nous montre que dans le beurre de cashcaval préparé en Roumanie il existe une plus grande proportion d'acides gras solubles que dans le beurre de brebis préparé par MARTIN.

4. L'indice de Reichert-Meissl, varie entre 27,43 et 31,57 , avec la moyenne de 29,88 .

5. L'indice de Polenske varie entre 4,25 et 5,3 avec la moyenne de 4,53 ce qui correspond aux résultats obtenus par d'autres auteurs. 
6. L'indice de $\mathrm{Hübl}$ (indice d'iode) varie entre 37,39 et 41,168 avec la moyenne de 39,43 , qui est supérieure à la valeur établie par d'autres auteurs.

La valeur de l'indice d'iode nous explique aussi la valeur inférieure du point de fusion et de solidification.

7. L'indice d'acidité est compris entre 0,35 et 1,62 (en moyenne, 1,13) ce qui est inférieur à la valeur établie par Peтkow qui, seul, a déterminé l'indice de l'acidité et trouvé une valeur de 2,01.

Chollet et Camus ont établi pour la graisse du lait de chèvre un rapport entre l'indice Reichert-Meissl d'une part et l'indice de Polenske, d'autre part, comme suit :

$$
100 \frac{\mathrm{P}}{\mathrm{R}-\mathrm{M}}=22,8-41,4
$$

tandis que pour la graisse du lait de vache, ce rapport varie entre 8 et 15 .

Si nous appliquons ce rapport au résultat de nos analyses, nous constatons qu'il varie entre 13,46 et 18,1 (avec la moyenne de $15,1)$ étant, en général, supérieur à celui établi pour la graisse du lait de vache, qui exceptionnellement arrive à 15 , et inférieur à celui établi pour la graisse du lait de chèvre.

\section{Conclusions}

De l'analyse des 8 échantillons de beurre de brebis (beurre de cashcaval) préparé en Roumanie et commercialisé au marché de la ville de Bucarest, on constate :

1. Le beurre de cashcaval provenant du lait de brebis, n'est mis dans le commerce que sous la forme de beurre fondu.

2. Le beurre de cashcaval ne contient plus que de la matière grasse. On lui ajoute rarement de petites quantités de $\mathrm{NaCl}$.

Par suite de sa composition, la valeur énergétique de ce beurre, pour cent, est supérieure à celui du lait de vache, qui contient aussi des substances non grasses.

3. La consistance du beurre de brebis peut être variable car, après fusion, la matière grasse n'est pas homogénéisée.

4. L'indice de Hehner est inférieur à celui établi par la majorité des auteurs, variant entre 85,46 et 86,9 (en moyenne 85,88 ), tandis que nous avons trouvé une valeur supérieure pour l'indice de Planchon 33,45 (en variant de 32,75 à 35,61 ) .

5. L'indice de Polenske est toujours supérieur à 4 , en variant de 4,25 à 5,3 (avec la moyenne de 4,53 ). Cet indice peut être utilisé pour établir les falsifications. 
6. Le rapport :

A. V.S.

A. V.I. $\times 100$, préconisé par Chollet et CAmus pour la graisse du lait de chèvre, varie, dans nos cas, entre 13,84 et 18,1 . Ce rapport est par conséquent supérieur à celui établi pour la graisse du lait de vache et inférieur à celui du lait de chèvre.

\section{BIBLIOGRAPHIE}

[1] PezzI. Staz. Spérim. Agrar. Ital., vol. XXVI, S. 615, 1894.

[2] N. Petkow. Z. Nahrungsmittel, Bd. 4, S. 826, 1901.

[3] R, Martin. Annales des Falsifications et des Fraudes, no 6, 1913.

[4] Sohmits. Cit. par Halden et Grun ([10] ei-dessous).

[5] Fasoetri et Savini. Congrès International de Laiterie, Paris, 16-19 mai 1926 , p. 329-332.

[6] O. LAxA. Revue Générale du Lait, nº 13-17, 1919.

[7] E. Darmors. Bull. Association des chimistes, 1937, 54.

[8] MaNgRANǴ et Femizat. Chimie analytique des huiles et graisses, 1933 p. 558 .

[9] Сноцlet et Camus. Annales des Falsifications et des Fraudes, 1938, p. 224.

[10] C. LOPEz. Journal Pharm. et Chim., 1937, p. 194.

[11] D. I. Garard et V. Pascate. Le Lait, 1937, I65.

[12] A. Bömer et W. Grossefeld. Handbuch der Lebensmittel Chemie, 1935 , Band 4.

[13] W. Halden et A. Grün. Analyse der Fätte und Wachse, 1929, Band 2, p. 480 .

[14] G. Génin. Le Lait, 1938, 174, 372.

[15] Rochajx et Tapernoux. Le lait et ses dérivés, Paris, 1942.

\section{LES PRODUITS IMILEI EN ALLEMICNE}

\section{par}

\section{A. CUÉNOT}

Ingénieur agronome, diplômé de la Section des Etudes supérieures des Industries du lait

Les renseignements qui sont donnés ci-dessous ont été rassemblés par une mission économique anglaise en Allemagne occupée. La découverte de l'organisation "Milei » et les investigations et constatations que fit cette mission. ont vivement intéressé les techniciens et économistes alliés.

Pour nous, Français, ces produits ne sont pas essentiellement nouveaux. Nous avons bien connu les protéines pendant les années 1943 et 1944, mais leur fabrication fut, à tort ou à raison, mal dirigée et leur commercialisation apporta en France beaucoup de 